\title{
PROBING AGN WITH WATER MASERS
}

J. M. MORAN, L. J. GREENHILL AND J. R. HERRNSTEIN Harvard-Smithsonian Center for Astrophyusics

60 Garden St., Cambridge MA 02138

P. J. DIAMOND

National Radio Astronomy Observatory

Socorro NM 86801

AND

M. MIYOSHI, N. NAKAI AND M. INOUE

National Astronomical Observatory

Minamisaku, Minamimaki, Nagano, 384-13, Japan

\section{Introduction}

Observations of the angular distribution of the water masers in the nucleus of NGC4258 reveal the presence of a thin molecular disk in nearly perfect Keplerian orbit (Miyoshi et al., 1995; Moran et al., 1995). About 300 galaxies have been searched for nuclear water masers to a limiting sensitivity of about $0.1 \mathrm{Jy}$ (e.g., Braatz, 1996); and 16 masers have been detected. The maser imaged by Miyoshi et al. (1995) offers the best example of disk structure. VLA data of NGC1068 shows evidence of disk structure (Gallimore et al., 1996; Greenhill \& Gwinn, 1996) and NGC2639 has drifting features, which may be due to centripetal acceleration (Wilson et al., 1995).

\section{Parameters of the Disk in NGC4258}

The morphology and kinematics of the molecular disk in the nucleus of NGC4258 are delineated by water masers, which have unresolved angular extents and narrow linewidths. The accurate definition of the disk properties were made with the Very Long Baseline Array (VLBA), which has both high angular resolution (0.2 mas at $1.35 \mathrm{~cm}$ wavelength) and high spectral resolution $\left(0.2 \mathrm{~km} / \mathrm{s}\right.$ or $\left.\nu / \delta \nu \sim 10^{6}\right)$. The high resolution image of the maser and its velocity field show that the disk is viewed nearly edge 


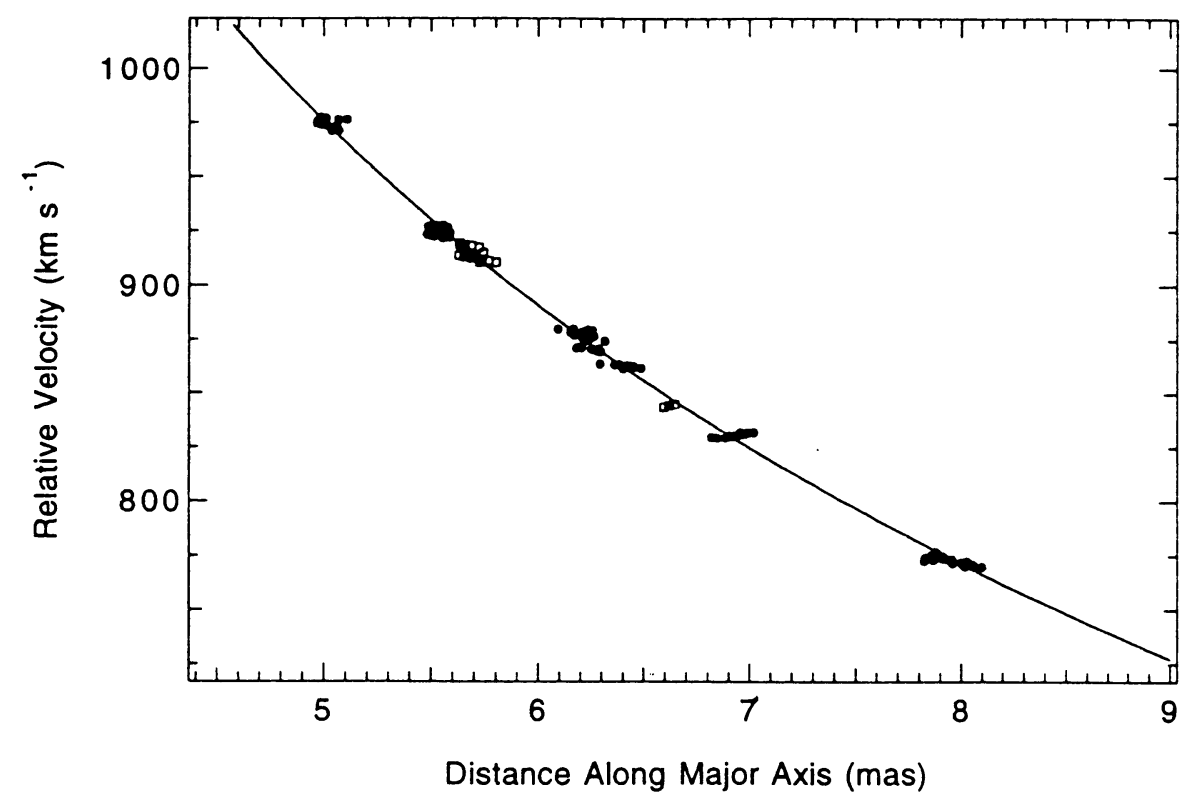

Figure 1. Keplerian rotation curve (magnitude of the line-of-sight velocity with respect to the systemic velocity versus radius from center) of the high velocity masers in the nucleus of NGC4258. Redshifted features, filled circles; blue shifted features, open squares.

on (inclination angle $=97^{\circ}$ ). The high-velocity features have a Keplerian distribution of velocities to an accuracy of better than 1 percent within an annulus of inner radius of $0.13 \mathrm{pc}(4$ mas and a distance of $6.4 \mathrm{Mpc}$ ) and outer radius of $0.26 \mathrm{pc}$ (see figure 1 ), which requires a binding mass of $3.5 \times 10^{7} \mathrm{M}_{\odot}$. The masers near the systemic velocity of the galaxy show a nearly linear dependence of line-of-sight velocity with impact parameter (apparent solid body rotation). The most reasonable explanation for these features is that they lie near the inner edge of the disk at a radius of about $0.13 \mathrm{pc}$ with a spread of about $0.01 \mathrm{pc}$.

The disk is slightly warped and its mean position angle is about $83^{\circ}$. The disk axis is parallel, at least in projection, with the synchrotron jet emerging from the nucleus, and inclined by $120^{\circ}$ to the rotation axis of the galaxy. Optical emission from the nucleus is linearly polarized in the direction parallel to the disk and may be due to Thomson scattering from electrons located along the spin axis of the disk (Wilkes et al., 1995).

The disk is very thin and we have not yet been able to measure its thickness. The systemic features have a vertical extent of $<0.01$ mas, and since their radius is about 4 mas, the ratio of height to radius is $<0.0025$. The disk is probably in hydrostatic equilibrium with a temperature of $<1000 \mathrm{~K}$ and a toroidal magnetic field of strength $<250 \mathrm{mG}$. The Toomre stability pa- 
rameter is probably in the range $1-10$, implying that the disk is marginally stable (Moran et al. 1995; Maoz, 1995). The inward drift velocity is less than $1 \mathrm{~m} / \mathrm{s}$ and the accretion rate is less that $10^{-3} \alpha \mathrm{M}_{\odot} \mathrm{y}^{-1}$, where $\alpha$ is the viscosity parameter. Detailed modelling (Neufeld \& Maloney, 1995) suggests that the accretion rate might be about $10^{-4} \alpha \mathrm{M}_{\odot} \mathrm{y}^{-1}$. The emission is extremely sub-Eddington $\left(L_{E}=4.5 \times 10^{45} \mathrm{erg} \mathrm{s}^{-1}, \mathrm{X}\right.$-Ray luminosity $=$ $4 \times 10^{40} \mathrm{erg} \mathrm{s}^{-1}$ ) (Lasota et al., 1996).

\section{Black hole}

The well defined Keplerian curve leaves little doubt that the gravitational binding mass is $3.5 \times 10^{7} \mathrm{M}_{\odot}$, which must be concentrated within a radius of $0.13 \mathrm{pc}$. The density of a spherical concentration of mass with that radius is $3.8 \times 10^{9} \mathrm{M}_{\odot} \mathrm{pc}^{-3}$ or $3 \times 10^{-13} \mathrm{~g} \mathrm{~cm}^{-3}$. Table 1 gives the minimuminferred central mass density for other massive black hole candidates.

TABLE 1. Galaxies with Possible Massive Black Holes

\begin{tabular}{lllllll}
\hline Galaxy & $\begin{array}{l}\text { Distance } \\
\text { Mpc }\end{array}$ & $\begin{array}{l}\text { Mass } \\
10^{6} \mathrm{M}_{\odot}\end{array}$ & $\begin{array}{l}\text { Diameter } \\
\mathrm{pc}\end{array}$ & $\begin{array}{l}\text { Density } \\
10^{6} \mathrm{M}_{\odot} \mathrm{pc}^{-3}\end{array}$ & $\mathrm{M} / \mathrm{L}$ & Ref \\
\hline Gal Center & 0.0085 & 2 & 0.3 & 90 & & 1 \\
M32 & 0.7 & 2 & 0.7 & 12 & & 2 \\
M31 & 0.7 & 30 & 0.7 & 180 & 100 & 2 \\
NGC4258 & 6.4 & 35 & 0.26 & 3800 & & 3 \\
NGC3115 & 8.4 & 1000 & 10 & 2 & 40 & 1 \\
NGC4594 & 9.2 & 500 & 18 & 0.2 & 100 & 1 \\
NGC3377 & 9.9 & 80 & 24 & 0.01 & 8 & 2 \\
M87 & 15 & 2400 & 36 & 0.1 & & 4 \\
NGC4261 & 30 & 1200 & 14 & 0.8 & 5200 & 5 \\
\hline
\end{tabular}

ref: (1) Genzel \& Harris (1994); (2) Kormendy \& Richstone (1995); (3) Miyoshi et al. (1995); (4) Harms et al. (1994); (5) Ferrarese et al. (1996)

\section{Centripetal Acceleration}

The features in the systemic group have long been known to drift in velocity. The interpretation of this phenomenon in terms of a rotating disk is clearly centripetal acceleration. Figure 2 shows spectra at two epochs. The high velocity features drift at less than $1 \mathrm{~km} \mathrm{~s}^{-1} \mathrm{y}^{-1}$, whereas the systemic features drift at about $9 \mathrm{~km} \mathrm{~s}^{-1} \mathrm{y}^{-1}$ (see, e.g., Greenhill et al. 1995). The amplitude variations and high spectral density of features in the systemic group make a precise estimate of their drifts difficult to estimate. 


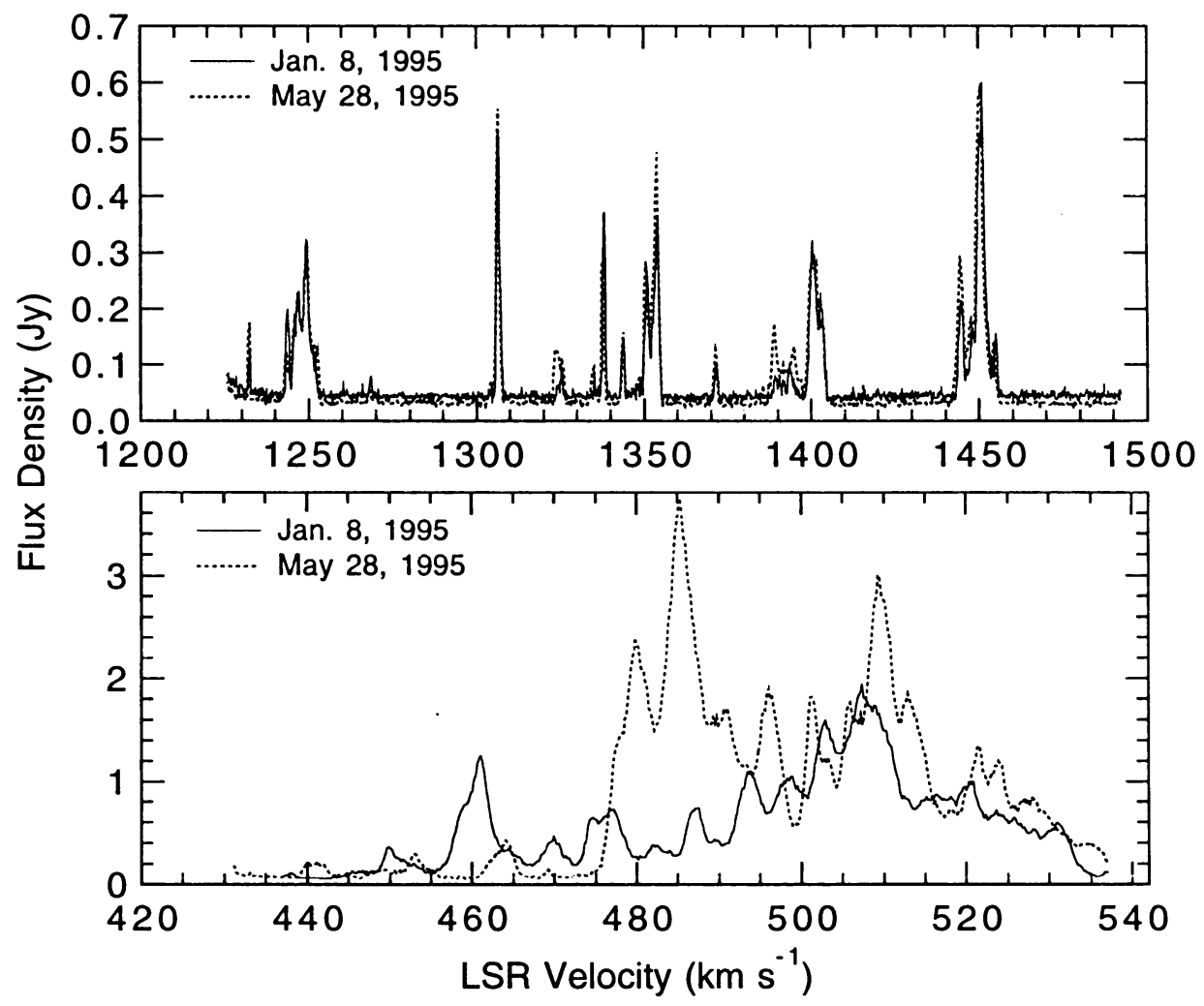

Figure 2. Centripetal acceleration evidenced by drifts in spectral features. (top) high velocity red-shifted features show less than $1 \mathrm{~km} \mathrm{~s}^{-1} \mathrm{y}^{-1}$ drift; (bottom) systemic velocity features drift at about $9 \mathrm{~km} \mathrm{~s}^{-1} \mathrm{y}^{-1}$, which is consistent with a distance of $6.4 \mathrm{Mpc}$

\section{References}

Braatz, J. (1996) PhD thesis, U. Maryland, in preparation.

Ferrarese, L., Ford, H., \& Jaffe, W. (1996) Astrophys. J., in press

Gallimore, J. F. et al. (1995) Astrophys. J. Lett., in press.

Genzel, R. \& Harris, A. I. (1994) The Nuclei of Normal Galaxies, Kluwer Academic Publishers, Dordrecht.

Greenhill, L. J. et al. (1995) Astron. Astrophys, 304, 21-33.

Harms, R. J. et al. (1994) Astrophys. J. Lett., 435, L35-L38.

Kormendy, J., and Richstone, D. (1995) Ann. Rev. A. \& A., 33, 581-624.

Lasota, J. P. et al. (1996) Astrophys. J., in press.

Maoz, E. (1995) Ap. J., 455, L131-L134.

Miyoshi, M., Moran, J., Herrnstein, J., Greenhill, L., Nakai, N., Diamond, P., Inoue, M. (1995) Nature, 373, 127-129.

Moran, J., Greenhill, L., Herrnstein, J., Diamond, P., Miyoshi, M., Nakai, N., and Inoue, M. (1995) Proc. Nat. Acad. Sci., 82, 5, 11427-11434.

Neufeld, D. A. \& Maloney, P. R. (1995) Astrophys. J., 447, L17-L20.

Wilkes, B. J., et al. (1995) A strophys. J., 455, L13-L16.

Wilson, A. S., Braatz, J. A., and Henkel, C. (1995) Astrophys. J. Lett., 455, L127-L129. 